\title{
AN INDEX THEOREM ON OPEN MANIFOLDS. I
}

\author{
JOHN ROE
}

\begin{abstract}
Introduction
This is the first of two papers which will describe and apply a new index theorem for elliptic operators on certain noncompact manifolds. This main result (Theorem 8.2) computes a real-valued index for operators of Dirac type on noncompact manifolds of "bounded geometry." In the sequel (Part II), several applications of this result will be given.

Let $D$ be a linear elliptic differential operator on a compact manifold $M$. Its kernel is then a finite-dimensional vector space of smooth functions, and one may try to compute the dimension of this space. This dimension depends rather sensitively on $D$, but the quantity

$$
\operatorname{Ind}(D)=\operatorname{dim}(\operatorname{ker} D)-\operatorname{dim}(\operatorname{coker} D)
$$

is a homotopy invariant of $D$, and the Atiyah-Singer index theorem [8], [4] calculates it from topological data.

Many analysts have produced generalizations of the index theorem to noncompact manifolds of various sorts, and these have been related to such diverse fields as the study of scalar curvature [23], number theory [5], representation theory [7], and the geometry of foliations [15]. Borrowing some terms from the theory of von Neumann algebras, we can roughly classify these results into three "types." Those of type I are nearest to the classical case. One imposes conditions sufficient to force the operator under consideration to be Fredholm in the usual sense, and the index is typically given by a formula similar to the usual one but with added correction terms. Under this heading could be included the $\eta$-invariant of Atiyah, Patodi, and Singer [6], the relative
\end{abstract}


index theorem described by Gromov and Lawson [23], and the result of [24]. For the type II theorems the operator is no longer Fredholm in the usual sense, but the index can nevertheless be interpreted as a real number by some kind of "averaging" or "renormalization" procedure. An early example was the index theorem for almost-periodic Toeplitz operators (Coburn, Douglas, Schaeffer, and Singer [13]), where the "averaging procedure" is in fact the Bohr mean value operator on almost-periodic functions; some other examples are the index theorems for coverings (Atiyah [3]; cf. Cheeger and Gromov [10]), for measured foliations (Connes [14]), and for homogeneous spaces of Lie groups (Connes and Moscovici [17]). Finally there are the type III theorems, where there is no averaging procedure and the index must be interpreted in a new way; the only example known to the author is the general index theorem for foliations (Connes and Skandalis [18]).

The theorem presented in this paper is of type II. Its basic structure may be set out diagrammatically as follows:

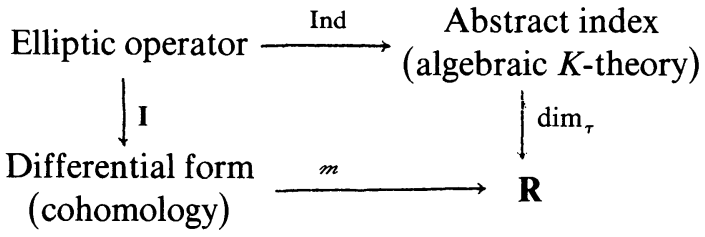

This sort of structure is spelt out explicitly in [15], and is in fact implicit in all the theorems of type II mentioned above.

We work on manifolds of bounded geometry - roughly speaking, of positive injectivity radius and bounded curvature; and on such manifolds we consider elliptic operators that are also of bounded geometry in a suitable sense. It is also necessary to impose some growth conditions on the solutions of elliptic equations, and in common with most other writers we work in the Hilbert space $L^{2}$.

On a manifold of bounded geometry it is natural to consider the cohomology of uniformly bounded differential forms. Using the Chern-Weil homomorphism we can make sense of the index class of an elliptic operator of bounded geometry as an element of this cohomology, and this defines the arrow I of the diagram above.

We can define on our manifold an algebra $\mathscr{U}_{-\infty}$ of uniformly smoothing operators, which as the name suggests are smoothing operators subject to certain uniform decay conditions. It turns out that an elliptic operator of bounded geometry has a parametrix modulo $\mathscr{U}_{-\infty}$, and its abstract index can therefore be defined as an element of the algebraic $K$-theory group $K\left(\mathscr{U}_{-\infty}\right)$. 
So far no averaging procedure has made its appearance. To obtain one we follow an idea introduced by Ahlfors [1], who defined a regular exhaustion of a noncompact manifold $M$ to be an increasing sequence $\left(M_{i}\right)$ of smoothly bounded open subsets of $M$ with the property that $\operatorname{Vol}\left(\partial M_{i}\right) / \operatorname{Vol}\left(M_{i}\right) \rightarrow 0$ as $i \rightarrow \infty$. A functional $m$ on bounded top-dimensional forms on $M$ can then be defined as the limit of the averaging functionals over the $M_{i}$; the regular exhaustibility condition ensures that $m$ passes to cohomology. Not all manifolds are regularly exhaustible; regular exhaustibility is an analogue for manifolds of amenability for groups (cf. Brooks [9]).

The elements of the algebra $\mathscr{U}_{-\infty}$ are represented by uniformly bounded smoothing kernels. Therefore, given a regular exhaustion, we can define a functional $\tau$ on $\mathscr{U}_{-\infty}$ by averaging kernels along the diagonal. The key point (Theorem 6.7) is that this functional is a trace, and therefore induces a dimension-homomorphism $\operatorname{dim}_{\tau}: K\left(\mathscr{U}_{-\infty}\right) \rightarrow \mathbf{R}$.

Our main theorem (Theorem 8.2) now says simply that the diagram commutes: $\operatorname{dim}_{\tau}(\operatorname{Int} D)=\langle\mathbf{I}(D), m\rangle$. To bring this down to earth we will briefly consider the example of the Riemann-Roch theorem in the plane, making use of the results of the sequel.

To obtain a type II Riemann-Roch theorem on $\mathbf{C}$, we define a divisor on $\mathbf{C}$ to be a finite formal linear combination of discrete subsets $\Gamma$ of $\mathbf{C}$, where the separation between the points of each $\Gamma$ is bounded below. It is possible to say what it means for a meromorphic function to be $L^{2}$-subordinate to such a divisor: it must be subordinate in the usual sense and square-integrable with respect to a standard weight function. When does such a divisor have nontrivial meromorphic functions $L^{2}$-subordinate to it?

It follows from Atiyah's index theorem for coverings [3] that if $\Gamma$ is a lattice in $\mathbf{C}$ then the divisor $[\Gamma]$ has meromorphic functions $L^{2}$-subordinate to it. (Notice that one obvious candidate-an elliptic function with poles on $\Gamma$-certainly is not square-integrable.) Using his index theorem for foliations, Connes [15] showed that if $\Gamma_{1}$ and $\Gamma_{2}$ are lattices in $\mathbf{C}$ with density $\left(\Gamma_{1}\right)>$ density $\left(\Gamma_{2}\right)$, then there are meromorphic functions $L^{2}$-subordinate to $\left[\Gamma_{1}\right]-$ $\left[z+\Gamma_{2}\right]$ for almost all translations $z$. In the sequel to this paper (Part II) it will be shown that there are meromorphic functions $L^{2}$-subordinate to any divisor of "uniformly positive" density. The theorem requires no periodic or almostperiodic structure, and consequently a much wider variety of divisors and deformations can be allowed.

Acknowledgement. I am indebted to Sir Michael Atiyah, Alain Connes, and Glenys Luke for helpful conversations and advice. In particular it will be clear to the reader that the theorem proved here is closely related to the results of [3] and [14]. The precise connections will be traced in Part II. I also thank the 
Science and Engineering Research Council of Great Britain and the Mathematical Sciences Research Institute of Berkeley for their support. Finally I should like to thank Carolyn Macqueen who translated [1] for me.

\section{Dirac-type operators}

When applying the Atiyah-Singer index theorem, one is usually interested only in elliptic operators related to some kind of geometrical structure, such as the de Rham, Dolbeault, or Dirac operators. It is well known that these may be subsumed under the title of "generalized Dirac operators"; in this paper we will restrict our attention to such operators. Here we review a few necessary facts about their analysis. More information may be found in [23].

Let $M$ be a Riemannian manifold, and let $\operatorname{Cliff}^{c}(M)$ be the complexified Clifford algebra bundle of $M$. Recall that $\operatorname{Cliff}^{c}(M)$ is equipped with a natural connection extending the Riemannian connection on $T M$, characterized by the relation

$$
\nabla\left(\phi_{1} \phi_{2}\right)=\phi_{1} \nabla \phi_{2}+\left(\nabla \phi_{1}\right) \phi_{2}
$$

for smooth sections $\phi_{1}$ and $\phi_{2}$ of $\operatorname{Cliff}^{c}(M)$.

Now suppose that $S$ is a bundle of left modules over the bundle of algebras Cliff $^{c}(M)$. We will say that $S$ is a Clifford bundle over $M$ if it is equipped with a hermitian metric and compatible connection such that:

(i) For each unit vector $e$ belonging to $T_{x}(M)$, the module multiplication $e: S_{x} \rightarrow S_{x}$ is an isometry;

(ii) For all smooth sections $\phi$ of $\operatorname{Cliff}^{c}(M)$ and $s$ of $S$, the compatibility relation $\nabla(\phi s)=\phi \nabla s+(\nabla \phi) s$ is satisfied.

If in addition $S$ is equipped with an involution $\eta$ anticommuting with the Clifford action of tangent vectors, then it will be called a graded Clifford bundle.

On a Clifford bundle $S$ there is a natural first-order differential operator $D=D_{s}$ called the Dirac operator. This is defined by the composition

$$
\Gamma(S) \rightarrow \Gamma\left(T^{*} M \otimes S\right) \rightarrow \Gamma(T M \otimes S) \rightarrow \Gamma(S) .
$$

Here the first arrow is given by the connection, the second by the metric, and the third by the Clifford module structure. In an orthonormal basis $\left\{e_{1}, \cdots, e_{n}\right\}$ for $T_{x} M$, one may write

$$
(D s)_{x}=\sum e_{k}\left(\nabla_{k} s\right)_{x}
$$

If $S$ is graded, then $D$ is also graded: it interchanges sections of the positive and negative eigenbundles of $\eta$. It is known (cf. [23]) that this definition 
encompasses all the "geometrical" operators to which the index theorem is usually applied.

Let $\langle$,$\rangle denote the L^{2}$ inner product on sections of $S$. Then

(1.1) Lemma. The Dirac operator is formally self-adjoint; any two smooth sections $s_{1}$ and $s_{2}$ of $S$, one of which is compactly supported, satisfy

$$
\left\langle D s_{1}, s_{2}\right\rangle=\left\langle s_{1}, D s_{2}\right\rangle \text {. }
$$

One can therefore ask about the possible self-adjoint extensions of $D$ as an unbounded operator on $L^{2}(S)$.

(1.2) Theorem. If the manifold $M$ is complete, then $D$ is essentially selfadjoint.

Thus, for any bounded Borel function $f$ on $\mathbf{R}$, the (bounded) operator $f(D)$ is uniquely defined by the spectral theorem. In particular, the one-parameter group $e^{i t D}$ is defined. The following property of this group will be of great importance.

(1.3) Theorem (unit propagation speed). Let $s$ be a compactly supported smooth section of $S$. Let $x$ be a point of $M$. Then $e^{i t D_{S}}$ is smooth and compactly supported, and $e^{i t D} s(x)=0$ for $|t|<\operatorname{distance}(x$, support $(s))$.

Proofs of (1.2) and (1.3) may be found for example in [12]; cf. also [32].

Finally, we will need the Weitzenbock formula for the Dirac operator. Let $\Omega: \wedge^{2} T^{*} M \rightarrow \operatorname{End}(S)$ denote the curvature operator of the connection on $S$, and let $R \in \operatorname{End}(S)$ be the operator described in an orthonormal basis $\left\{e_{i}\right\}$ by

$$
R s=\frac{1}{2} \sum e_{i} e_{j} \Omega\left(e_{i} \wedge e_{j}\right) s
$$

Then one has

(1.4) Lemma. For any smooth section $s$ of $S$,

$$
D^{2} s=\nabla^{*} \nabla s+R s .
$$

\section{Bounded geometry}

This section will summarize some of the analytical properties of Dirac operators on noncompact manifolds. We are interested in uniform estimates, and to get these we need conditions of "bounded geometry" (cf. [10], [11], [20]).

(2.1) Definition. Let $M$ be a complete Riemannian manifold. It will be said to have bounded geometry if

(i) $M$ has positive injectivity radius, and

(ii) The curvature tensor of $M$ is uniformly bounded, as are all its covariant derivatives. 
A Clifford bundle $S$ over $M$ will be said to have bounded geometry if

(iii) The curvature tensor of $S$ is uniformly bounded, as are all its covariant derivatives.

Examples. Any compact manifold has bounded geometry; more generally, any manifold arising as a leaf in a foliation of a compact manifold, or as an unramified covering of a compact manifold, has bounded geometry. A compactly supported perturbation-for example, a connected sum-preserves bounded geometry. Any noncompact manifold of bounded geometry has infinite volume.

Now let $M$ be a complete $n$-dimensional Riemannian manifold. Recall that a normal coordinate system near $m \in M$ is a coordinate chart near $m$ defined by a choice of orthonormal frame for $T_{m} M$ together with a restriction of the corresponding exponential map $\exp _{m}: \mathbf{R}^{n} \rightarrow M$ to some neighborhood $B$ of the origin on which it is a diffeomorphism. This coordinate system gives rise to a framing of the tangent bundle near $m$, but this framing may not be orthonormal. To measure its derivation from orthonormality we define the distortion of the coordinate system to be the supremum of

$$
\operatorname{Max}\left(\sup \left|\left(\exp _{m}\right)_{*} X\right|, \sup \left|\left(\exp _{m}\right)_{*} X\right|^{-1}\right)
$$

as $X$ ranges over unit vector fields on $B$.

(2.2) Lemma. Suppose that $M$ is a Riemannian n-manifold of bounded geometry. Then there is a ball $B$ with center 0 in $\mathbf{R}^{n}$ such that:

(i) $B$ is the domain of a normal coordinate system at every point $m$ of $M$;

(ii) the distortion of the normal coordinate system on $B$ is bounded independently of $m$.

Proof. This is an easy consequence of the Rauch comparison theorem [28, volume II, p. 76].

(2.3) Remark. If $M$ is oriented, it also follows from the comparison theorem that the volume of a ball of radius $r$ in $M$ is at most equal to the volume of a ball of radius $r$ in a hyperbolic space of constant curvature everywhere less than that of $M$.

In normal coordinates, the connection on a Riemannian manifold can be expressed by Christoffel symbols $\Gamma_{i j}^{k}$. Bounded geometry translates into a uniform boundedness condition on these Christoffel symbols:

(2.4) Proposition. A Riemannian $n$-manifold $M$ has bounded geometry if and only if there is a ball $B$ with center 0 in $\mathbf{R}^{n}$ such that

(i) $B$ is the domain of a normal coordinate system at every point $m$ of $M$;

(ii) the Christoffel symbols of $M$, considered as a family of smooth functions parametrized by indices $i, j$, and $k$ and by a point $m$ of $M$, lie in a bounded subset of the Fréchet space $C^{\infty}(B)$. 
For the proof, one can integrate the well-known differential equations expressing the curvature in terms of the connection coefficients, [4, Appendix].

Such a ball $B$ will be called a good coordinate ball.

Suppose now that $S$ is a Clifford bundle over $M$. If we choose an orthonormal frame for $S$ at a point $m$ of $M$, then we may extend it to a framing for $S$ on the domain of a normal coordinate system near $m$ by parallel translation along radial geodesics. Such a framing is called synchronous; we will also speak loosely of "normal coordinates" for $S$. With respect to such a framing, the connection on $S$ may be expressed in terms of Christoffel symbols $\Gamma_{i \alpha}^{\beta}$.

(2.5) Proposition. Let $M$ be a manifold of bounded geometry. A Clifford bundle $S$ over $M$ has bounded geometry if and only if there is a good coordinate ball $B$ such that the Christoffel symbols for $S$ lie in a bounded subset of $C^{\infty}(B)$.

Proof. Similar to (2.4).

Where appropriate, the term "good coordinate ball" will be taken to include this condition also.

For the rest of this section, $M$ will be a Riemannian $n$-manifold of bounded geometry and $S$ a Clifford bundle of bounded geometry over it, with corresponding Dirac operator $D$.

(2.6) Definition. Let $k$ be a nonnegative integer. The Sobolev space $W^{k}(S)$ is the completion of $C_{c}^{\infty}(S)$ in the norm

$$
\|s\|_{k}=\left(\|s\|^{2}+\|D s\|^{2}+\cdots+\left\|D^{k} s\right\|^{2}\right)^{1 / 2} \text {. }
$$

For negative $k, W^{k}(S)$ is the dual space of $W^{-k}(S)$, regarded as a space of distributional sections of $S$. Also, $W^{\infty}(S)=\cap W^{k}(S)$ and $W^{-\infty}(S)=$ $\cup W^{k}(S) ; W^{\infty}(S)$ is equipped with the obvious Fréchet topology, and $W^{-\infty}(S)$ is equipped with the weak topology that it inherits as the dual of $W^{\infty}(S)$.

(2.7) Definition. Let $r$ be a nonnegative integer. The uniform $C^{r}$ space $U C^{r}(S)$ is the Banach space of all $C^{r}$ sections $s$ of $S$ such that the norm

$$
\|\| s\|\|_{r}=\sup \left\{\left|\nabla_{n_{1}} \cdots \nabla_{r_{4}} s(m)\right|\right\}
$$

is finite, where the supremum is taken over all $m \in M$ and all collections $v_{1}, \cdots, v_{q}(0 \leqslant q \leqslant r)$ of unit tangent vectors at $m$. Also, $U C^{\infty}(S)$ will denote the Fréchet space $\cap U C^{r}(S)$.

(2.8) Proposition (Sobolev embedding). The Fréchet space $W^{\infty}(S)$ is continuously included in $U C^{\infty}(S)$.

Proof. This follows, as on a compact manifold, from the elliptic estimate for $D$. Since by (2.4) the coefficients and ellipticity constant of $D$ written in local coordinates in a good ball are uniformly bounded, the norms of the local Sobolev embeddings are also uniformly bounded, which gives the result. 
(2.9) Proposition. A continuous linear operator from $W^{-\infty}(S)$ to $W^{\infty}(S)$ is represented by a smoothing kernel which is uniformly bounded, as are all its covariant derivatives. The map

$$
L\left(W^{-\infty}(S), W^{\infty}(S)\right) \rightarrow U C^{\infty}(S \otimes S)
$$

which associates its kernel to such an operator is continuous (where $L\left(W^{-\infty}(S), W^{\infty}(S)\right)$ is equipped with the topology of bounded convergence).

Proof. Similar.

Let $\mathscr{R}(\mathbf{R})$ denote the Fréchet space of rapidly decaying functions on $\mathbf{R}$, that is the space of continuous functions $f$ such that for each $k$

$$
|f(x)| \leqslant C_{k}(1+|x|)^{-k}
$$

with the best constants $C_{k}$ as seminorms. Clearly the usual Schwartz space $\mathscr{S}(\mathbf{R})$ is continuously included in $\mathscr{R}(\mathbf{R})$.

(2.10) Proposition. Let $f \in \mathscr{R}(\mathbf{R})$. Then the operator $f(D)$ (defined by the spectral theorem) is represented by a smoothing kernel which is uniformly bounded, as are all its covariant derivatives. The map

$$
\mathscr{R}(\mathbf{R}) \rightarrow U C^{\infty}(S \otimes S)
$$

which associates its kernel to such an operator is continuous.

Proof. Immediate.

In the analytical proof of the index theorem on compact manifolds [4], a crucial part is played by an asymptotic expansion for the fundamental solution of the "heat equation" $\partial s / \partial t+D^{2} s=0$. We shall need the analogous expansion on a noncompact manifold of bounded geometry.

(2.11) Proposition. Let $M, S$, and $D$ be as above, with $M$ oriented. Then the operator $e^{-t D^{2}}$ is represented by a uniformly bounded smoothing kernel $k_{t}(x, y)$, and there is an asymptotic expansion

$$
k_{t}(x, x) \sim \sum t^{(k-n) / 2} \Psi_{k}(x)
$$

where the $\Psi_{k}$ are smooth sections of $\operatorname{End}(S) \otimes \wedge^{n} T^{*} M$, locally computable in terms of the curvatures of $M$ and $S$ and their covariant derivatives. Moreover, the remainder terms implicit in the asymptotic expansion are uniformly bounded in $x$.

The proof is essentially the same as the classical one on compact manifolds, for which see Gilkey [21].

If $S$ has a grading $\eta$, we define the index-form $\mathbf{I}(D)$ to be the $n$-form $\operatorname{Trace}\left(\eta \Psi_{n}\right)$. It is a local invariant of the Clifford bundle structure, and so the calculations of it made in [4] for the case of a compact manifold remain valid. These calculations show that for all the classical operators the index-form corresponds under the Chern-Weil homomorphism to the top-dimensional part of the index class

$$
(-1)^{n} \operatorname{ch}(\sigma(D)) \cup t d(T M \otimes \mathbf{C})
$$


which appears in the Atiyah-Singer index formula [8]. Here $D$ is considered as an elliptic operator between the +1 and -1 eigenbundles of the grading $\eta$.

\section{Bounded Chern-Weil theory}

In this section we discuss the Chern-Weil theory of noncompact manifolds of bounded geometry. Similar ideas are developed further in [27].

Let $M$ be a complete, oriented Riemannian manifold, and let $\Omega_{\beta}^{p}$ denote the Banach space of $p$-forms on $M$ which are bounded in the norm

$$
\|\alpha\|=\sup \{|\alpha(x)|+|d \alpha(x)|: x \in M\} .
$$

Then there is a complex of Banach spaces and continuous linear maps:

$$
\Omega_{\beta}^{0} \stackrel{d}{\rightarrow} \Omega_{\beta}^{1} \stackrel{d}{\rightarrow} \cdots \stackrel{d}{\rightarrow} \Omega_{\beta}^{n} .
$$

(3.1) Definition. The $\beta$-cohomology groups of $M$ are defined to be the groups

$$
H_{\beta}^{p}(M)=\left[\operatorname{Ker} d_{p}\right] /\left[\text { Closure of } \operatorname{Im} d_{p-1}\right]
$$

We shall mostly be concerned with the top-dimensional $\beta$-cohomology group; but it is convenient to consider the others as well.

(3.2) Definition [31]. The manifold $M$ is closed at infinity if for any continuous function $\lambda$ on $M$ such that $0<K^{-1}<\lambda<K$ for some constant $K$, the form $\lambda$. vol gives rise to a nontrivial cohomology class in $H_{\beta}^{n}(M)$.

(3.3) Definition. A fundamental class for $M$ is a positive linear functional $m: \Omega_{\beta}^{n} \rightarrow \mathbf{R}$ such that $\langle\operatorname{vol}, m\rangle \neq 0$ and $m \circ d=0$.

It follows from the Hahn-Banach theorem that $M$ has a fundamental class if and only if it is closed at infinity.

(3.4) Examples. A compact manifold is closed at infinity, and its $\beta$ cohomology coincides with the ordinary cohomology. Euclidean $n$-space is closed at infinity. However, hyperbolic $n$-space is not closed at infinity, for its volume form is the exterior derivative of a bounded ( $n-1)$-form.

We will now review some of the basic concepts of Chern-Weil theory, as set out in $[28, \S \mathrm{XII}]$. Let $G$ be a compact Lie group with Lie algebra $g$. Let $I(G)$ denote the graded subalgebra of the symmetric algebra on $g^{*}$ consisting of mappings $f: g \times g \times \cdots \times g \rightarrow \mathbf{R}$ which are invariant under the adjoint action of $G$. Let $P$ be a principal $G$-bundle over a manifold $M$, and let $R_{g}$ denote right translation by $g$ on $P$. 
(3.5) Lemma. Let $\alpha$ be an arbitrary $p$-form on P. If $\alpha$ is horizontal and

$$
R_{g}^{*} \alpha=\alpha \quad \forall g \in G,
$$

then $\alpha$ is the pull-back to $P$ of a uniquely determined $p$-form $\alpha^{\#}$ on $M$.

Proof. [28, vol. II, p. 294].

Suppose now that $P$ is equipped with a connection; this is described by a $g$-valued 1-form $\omega$ of the adjoint type on $P$. Its curvature is the exterior covariant derivative $\Omega=D \omega$, which is a horizontal 2-form of the adjoint type. Let $f \in I^{p}(G)$. Given differential forms $\alpha_{1}, \cdots, \alpha_{p}$ on $P$, with values in $g$, one may construct a form $f\left(\alpha_{1}, \cdots, \alpha_{p}\right)$ with values in $\mathbf{R}$, by a straightforward antisymmetrization procedure. If the forms $\alpha_{1}, \cdots, \alpha_{p}$ are of the adjoint type, then $f\left(\alpha_{1}, \cdots, \alpha_{p}\right)$ will be invariant under right translation; if in addition the forms $\alpha_{1}, \cdots, \alpha_{p}$ are horizontal, then $f\left(\alpha_{1}, \cdots, \alpha_{p}\right)$ will by (3.5) be the pull-back of a form on $M$.

(3.6) Theorem (Chern-Weil). Let $P$ be a principal bundle over $M$ with structural group $G$; let $f \in I^{k}(G)$. Then:

(i) For any connection $\omega$ in $P$ with curvature $\Omega$, the $2 k$-form $f(\Omega, \cdots, \Omega)^{\#}$ on $M$ is closed;

(ii) For a fixed $\Omega$, the map $f \rightarrow f(\Omega, \cdots, \Omega)^{\#}$ is a homomorphism of $I(G)$ into the algebra of differential forms on $M$;

(iii) Suppose that $\omega_{0}$ and $\omega_{1}$ are two connections on $M, \omega_{t}=(1-t) \omega_{0}+t \omega_{1}$, $\Omega_{t}$ the curvature of $\omega_{t}$. Then

$$
f\left(\Omega_{0}, \cdots, \Omega_{0}\right)^{\#}-f\left(\Omega_{1}, \cdots, \Omega_{1}\right)^{\#}=d \Phi^{\#},
$$

where

$$
\Phi=k \int_{[0,1]} f\left(\omega_{1}-\omega_{0}, \Omega_{t}, \cdots, \Omega_{t}\right) d t
$$

Proof. [28, loc.cit., pp. 293-297].

Notice that the difference $\omega_{1}-\omega_{0}$ of two connections is a horizontal 1-form of the adjoint type.

Suppose further that $M$ is equipped with a Riemannian metric. Let $\pi$ denote the projection of the principal bundle $P$ onto $M$, and equip $g$ with an inner product. A horizontal $p$-form $\alpha$ of the adjoint type will be called bounded if there is a constant $C$ such that

$$
\left|\alpha\left(h_{1} \wedge \cdots \wedge h_{p}\right)\right| \leqslant C\left|\pi_{*}\left(h_{1} \wedge \cdots \wedge h_{p}\right)\right| .
$$

Here the norm on the left is given by the inner product on $g$ and that on the right by the Riemannian metric. 
(3.7) Definition. We will say that a connection on $P$ has bounded curvature if its curvature form is bounded in the sense described above. If $\omega_{0}$ and $\omega_{1}$ are connections with bounded curvature, and $\omega_{1}-\omega_{0}$ is bounded, we will say that $\omega_{0}$ and $\omega_{1}$ are boundedly equivalent.

Now one has the following version of the Chern-Weil theorem.

(3.8) Theorem. Let $P$ be a principal G-bundle over the Riemannian manifold $M$. Then for any connection of bounded curvature $\Omega$ on $P$, there is a ring homomorphism $I(G) \rightarrow H_{\beta}^{*}(M)$ defined by $f \rightarrow\left[f(\Omega, \cdots, \Omega)^{\#}\right]$. Moreover, this homomorphism depends only on the bounded equivalence class of the connection.

Proof. Immediate from (3.6).

From the standard presentations of Chern-Weil theory one knows in fact that for any compact Lie group $G, I(G)$ is isomorphic to the real cohomology of the classifying space of $G$. In particular, for $G=\operatorname{SO}(n)$, the ring $I(G)$ is generated by the Pontrjagin and Euler classes.

Now let $M$ be an oriented Riemannian manifold of bounded geometry. Associated to the tangent bundle is the principal $\operatorname{SO}(n)$-bundle of oriented orthonormal frames on $M$, and the Levi-Civita connection on this bundle clearly has bounded curvature. Thus the Pontrjagin and Euler classes of $M$ are defined as elements of $H_{\beta}^{*}(M)$. Similarly, if $S$ is a (Clifford) bundle of bounded geometry on $M$, then its Chern classes are defined as elements of $H_{\beta}^{*}(M)$. In particular the index form $\mathbf{I}(D)$ for a classical Dirac operator $D$ is an element of $H_{\beta}^{n}(M)$.

Let $f: M \rightarrow M^{\prime}$ be a smooth map between Riemannian manifolds. One says that $f$ has bounded dilatation if there is a constant $C$ such that $\left|f_{*} v\right| \leqslant C|v|$ for all $v \in T M$. Such an $f$ induces a map on $\beta$-cohomology. If $f$ is a diffeomorphism, and both $f$ and $f^{-1}$ have bounded dilatation, then $f$ is called a quasi-isometry, and it induces an isomorphism on $\beta$-cohomology. Finally, $f$ is called a strict quasi-isometry if it is a quasi-isometry and the Levi-Civita connection on $M$ is equivalent in the sense of (3.7) to the pull-back of the Levi-Civita connection on $M^{\prime}$. Notice that if $M$ is a compact manifold, a leaf in a foliation of a compact manifold, or a covering of a compact manifold, then it has a Riemannian metric of bounded geometry that is unique up to strict quasi-isometry.

(3.9) Lemma. Let $M$ and $M^{\prime}$ be Riemannian manifolds of bounded geometry, with $f: M \rightarrow M^{\prime}$ a strict quasi-isometry. Let $m$ be a fundamental class for $M$, and let $\sigma$ be an element of $H_{\beta}^{*}\left(M^{\prime}\right)$. Then for any SO characteristic class $c$,

$$
\left\langle c(M) \cup f^{*} \sigma, m\right\rangle=\left\langle c\left(M^{\prime}\right) \cup \sigma, f_{*} m\right\rangle .
$$

Proof. Obvious. 


\section{Abstract indices}

Let $A$ be an algebra with unit over some field $k$, and let $B$ be an ideal in $A$. Abstractly, an "elliptic operator" is an element of $A$ which is invertible modulo the algebra $B$ of "lower order terms"; for example, $A$ could be the algebra of bounded operators on the $L^{2}$ space of a compact manifold, and $B$ could be the ideal of compact or of smoothing operators. Such an elliptic operator defines an element of the algebraic $K$-theory group $K_{1}(A / B)$, and its index is defined to be the element of $K(B)=K_{0}(B)$ corresponding to this under the connecting homomorphism of the long exact sequence in algebraic $K$-theory.

A slightly more general concept of "abstract elliptic operator" will in fact be needed, which would correspond classically to the extension to operators not on functions but on sections of vector-bundles. Let $M$ and $N$ be finite projective right $A$-modules, and let $P: M \rightarrow N$ be an $A$-linear map. It will be called abstractly elliptic if there is a "parametrix" $Q: N \rightarrow M$ such that

$$
\operatorname{Im}(Q P-1) \subset M \otimes B, \quad \operatorname{Im}(P Q-1) \subset N \otimes B ;
$$

equivalently, $P$ is abstractly elliptic if it becomes an isomorphism on tensoring with $A / B$.

Form the Cartesian square of algebra homomorphisms

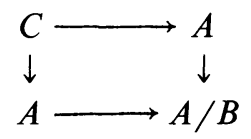

where $C$ is the double of $A$ along $B$ [29, \$4]. Correspondingly, let the $C$-module $X$ be the product of $M$ and $N$ over the isomorphism $P \otimes(A / B)$. By the results of $[29, \S 2], X$ is a finite projective $C$-module and so gives rise to an element of $K(C)$. Now there is a split short exact sequence

$$
0 \rightarrow B \rightarrow C \rightarrow A \rightarrow 0
$$

so that $K(C) \simeq K(B) \oplus K(A)$; we define $\operatorname{Ind}(P)$ to be the $K(B)$ component of the class $[X]$ in $K(C)$. Notice that this definition is natural in the sense that if $i: A \rightarrow A^{\prime}$ is a homomorphism mapping $B$ into $B^{\prime}$, then $\operatorname{Ind}\left(i_{*} P\right)=$ $i_{*}($ Ind $P$ ).

A $k$-linear functional $\tau$ on $B$ is called a trace if $\tau\left(b_{1} b_{2}\right)=\tau\left(b_{2} b_{1}\right)$ for all $b_{1}$, $b_{2}$ in $B$. For a projective $A$-module $M$, let $\operatorname{End}_{B}(M)$ denote the algebra of $A$-linear endomorphisms of $M$ that map $M$ to $M \otimes B$. Then $\tau$ extends to a trace on $\operatorname{End}_{B}(M)$ : if $M \simeq A^{r}$ is free, then $\operatorname{End}_{B}(M)$ is isomorphic to the matrix algebra $\operatorname{Mat}_{r}(B)$, and $\tau$ may be extended by tensoring (over $k$ ) with the usual trace on $\operatorname{Mat}_{r}(k)$; a general $M$ may be embedded in a free module. 
The trace $\tau$ may be extended to the algebra $B^{+}$, obtained by adjunction of a unit to $B$, if one sets $\tau(1)=0$. It is well known that the trace on idempotents of $\operatorname{Mat}\left(B^{+}\right)$passes to equivalence classes and therefore extends to a "dimension homomorphism" $\operatorname{dim}_{\tau}: K(B) \rightarrow k$.

(4.1) Lemma. If $P$ is an abstract elliptic operator with parametrix $Q$, then

$$
\operatorname{dim}_{\tau}(\text { Ind } P)=\tau(1-Q P)^{2}-\tau(1-P Q)^{2} .
$$

This result is certainly known, and the particular case where $\tau$ is the ordinary Hilbert-space trace is proven, for example, in [25, Lemma 7.1]; but the author has not found this version in print.

Proof. We do the special case $M=N=A$; then $P$ and $Q$ are elements of $A$. Let $R$ be the element

$$
\left[\begin{array}{cc}
(2-P Q) P & P Q-1 \\
1-Q P & Q
\end{array}\right]
$$

of $\operatorname{Mat}_{2}(A)$. It is invertible, its inverse being

$$
\left[\begin{array}{cc}
Q & 1-Q P \\
P Q-1 & (2-P Q) P
\end{array}\right]
$$

and it is equal modulo $B$ to

$$
\left[\begin{array}{ll}
P & 0 \\
0 & Q
\end{array}\right]
$$

The construction of $[29, \S 2]$ shows that the module $X$ is described by the projection $\left(\pi, R \pi R^{-1}\right)$ in $\operatorname{Mat}_{2}(C)$, where $\pi \in \operatorname{Mat}_{2}(A)$ is the projection onto the first factor. A calculation shows that

$$
R \pi R^{-1}=\left[\begin{array}{cc}
1-(1-P Q)^{2} & * \\
* & (1-Q P)^{2}
\end{array}\right]
$$

and the result follows.

This formula reduces to the more familiar one

$$
\tau(1-Q P)-\tau(1-P Q)
$$

if $\tau$ enjoys the additional property that $\tau(a b)=\tau(b a)$ for all $a$ in $A$ and $b$ in $B$.

We record for future reference the following well-known fact.

(4.2) Lemma. The inclusion of $B$ in $\operatorname{Mat}_{r}(B)$ by the top left corner induces an isomorphism between $K(B)$ and $K\left(\operatorname{Mat}_{r}(B)\right)$. 


\section{Uniform operators}

Let $M$ be an oriented, connected, noncompact Riemannian manifold of bounded geometry. The following notation will be used without further comment:

$d\left(x, x^{\prime}\right)$ is the distance between points $x, x^{\prime} \in M$;

$B(x, r)$ is the open ball with center $x$ and radius $r$;

$B^{\prime}(x, r)$ is the closure of $B(x, r)$;

$\operatorname{Pen}^{+}(K, r)$ is the closure of $\bigcup\{B(x, r): x \in K\}$;

$\operatorname{Pen}^{-}(K, r)$ is the closure of $M \backslash \operatorname{Pen}^{+}(M \backslash K, r)$.

("Pen" is an abbreviation for "penumbra.") The following geometrical fact will be important both here and in the sequel.

(5.1) Proposition. There are monotone increasing functions $V_{0}$ and $V_{1}$ : $\mathbf{R}^{+} \rightarrow \mathbf{R}^{+}$such that

(i) $V_{0}(r) \rightarrow \infty$ as $r \rightarrow \infty$;

(ii) $V_{1}(r) \rightarrow 0$ as $r \rightarrow 0$;

(iii) $V_{0}(r)>0 \forall r>0$;

(iv) $V_{0}(r) \leqslant \operatorname{Vol} B(x, r) \leqslant V_{1}(r) \forall x \in M$.

Proof. Straightforward from (2.2) and (2.3).

Let $S$ be a Clifford bundle on $M$. Recall (2.8) that $W^{k}(S)$ is the $k$ th global Sobolev space of sections of $S$, with norm \|\|$_{k}$. If $L$ is a subset of $M$, then $\|f\|_{k, L}$, will denote the infimum of $\left\{\|g\|_{k}: g \in W^{k}(S), g=f\right.$ on a neighborhood of $L\}$. It is clear that \|\|$_{k, L}$ is a continuous seminorm on $W^{k}$.

Let $S$ and $S^{\prime}$ be Clifford bundles over $M$, and let $A$ be an operator mapping $W^{k}(S)$ to $W^{l}\left(S^{\prime}\right)$. We will say that $A$ maps $W^{k}$ to $W^{l}$ quasilocally if there is a function $\mu: \mathbf{R}^{+} \rightarrow \mathbf{R}^{+}$such that $\mu(r) \rightarrow 0$ as $r \rightarrow \infty$ and such that for each $K \subset M$ and each $u \in W^{k}(S)$ supported within $K$,

$$
\|A u\|_{l, M \backslash \operatorname{Pen}^{+}(K, r)} \leqslant \mu(r)\|u\|_{k},
$$

$\mu$ will be called a dominating function for $A$.

We will also speak of quasilocal operators in case one or both of the bundles $S$ and $S^{\prime}$ are trivial. To do this, regard the trivial bundle $M \times \mathbf{C}^{k}$ as a subbundle of the Clifford bundle $\wedge^{*} T^{*} M \otimes C^{k}$.

(5.2) Proposition. If $A$ maps $W^{k}$ to $W^{l}$ quasilocally, and $B$ maps $W^{l}$ to $W^{m}$ quasilocally, then $B A$ maps $W^{k}$ to $W^{m}$ quasilocally.

Proof. Let $\mu_{A}$ and $\mu_{B}$ be dominating functions for $A$ and $B$ respectively, and let $u \in W^{k}$ be supported within $K$. Let $L=M \backslash \operatorname{Pen}^{+}(K, r / 2)$. By definition, there is $g \in W^{l}$ coinciding with $A u$ on $L$ and such that $\|g\|_{l} \leqslant$ $2 \mu_{A}(r / 2)\|u\|_{k}$. Therefore

$$
\begin{aligned}
\|B A u\|_{m, M \backslash \mathrm{Pen}^{+}(K, r)} & \leqslant\|B g\|_{m}+\|B(A u-g)\|_{m, M \backslash \mathrm{Pen}^{\prime}(K, r)} \\
& \leqslant\left(\|B\| \cdot 2 \mu_{A}(r / 2)+\mu_{B}(r / 2)\left(\|A\|+2 \mu_{A}(r / 2)\right)\right)\|u\|_{k} .
\end{aligned}
$$


The expression enclosed within the outer brackets is a dominating function for $B A$.

(5.3) Definition. An operator $A: C_{c}^{\infty}(S) \rightarrow C^{\infty}\left(S^{\prime}\right)$ is called a uniform operator of order $\leqslant k$ if for each $r$ it has a continuous extension to a quasilocal operator from $W^{r}(S)$ to $W^{r-k}\left(S^{\prime}\right)$. If $S=S^{\prime}$, the collection of uniform operators of order $\leqslant k$ will be denoted $\mathscr{U}_{k}(S)$, or $\mathscr{U}_{k}(M)$ in case $S$ is a 1-dimensional trivial bundle.

It is clear that the uniform operators on a bundle $S$ form a filtered algebra $\mathscr{U}(S)$, in which the uniform operators of order $-\infty$ form an ideal $\mathscr{U}_{-\infty}(S)=$ $\cap \mathcal{U}_{k}(S)$.

(5.4) Proposition. Let $A \in \mathscr{U}_{-\infty}(M)$ be a uniform operator of order $-\infty$ acting on functions on $M$. Then $A$ is represented by a uniformly bounded smoothing kernel $k(x, y)$ :

$$
A u(x)=\int k(x, y) u(y) \cdot \operatorname{vol}(y)
$$

Moreover there is a function $\nu=\nu(r)$ tending to zero as $r \rightarrow \infty$ such that

$$
\int_{M \backslash B(x, r)}|k(x, y)|^{2} \cdot \operatorname{vol}(y)<\nu(r) ; \int_{M \backslash B(x, r)}|k(y, x)|^{2} \cdot \operatorname{vol}(y)<\nu(r) .
$$

Proof. By (2.9), $A$ is a smoothing operator with uniformly bounded kernel. Now, let $\varepsilon_{x}$ denote the Dirac mass at $x$; from (2.8), there is $k$ such that $\varepsilon_{x}$ belongs to $W^{-k}(M)$ with norm bounded by some constant $C$ independent of $x$. Since $A$ maps $W^{-k}(M)$ quasilocally to $W^{0}(M)$, there is a dominating function $\mu$ such that

$$
\begin{aligned}
\int_{M \backslash B(x, r)}|k(y, x)|^{2} \cdot \operatorname{vol}(y) & =\left\|A \varepsilon_{x}\right\|_{0, M \backslash B(x, r)}^{2} \\
& \leqslant \mu(r)^{2}\left\|\varepsilon_{x}\right\|^{2} \leqslant(C \mu(r))^{2} .
\end{aligned}
$$

Since $A$ maps $W^{0}(M)$ quasilocally to $W^{\infty}(M)$, which is continuously included in $U C^{\infty}(M)$, there is a dominating function $\mu^{\prime}$ such that for all squareintegrable $u$ on $M$ supported outwith $B(x, r)$,

$$
\left|\int_{M} k(x, y) u(y) \operatorname{vol}(y)\right| \leqslant \mu^{\prime}(r)\|u\|_{0} .
$$

This implies that

$$
\int_{M \backslash B(x, r)}|k(x, y)|^{2} \cdot \operatorname{vol}(y) \leqslant\left(\mu^{\prime}(r)\right)^{2} .
$$

The proof is completed. 
The space $S^{m}(\mathbf{R})$ of symbols of order $\leqslant m$ on $\mathbf{R}$ is defined to be the subspace of $C^{\infty}(\mathbf{R})$ consisting of functions $f$ satisfying estimates of the form

$$
\left|f^{(k)}(\lambda)\right|<C_{k}(1+|\lambda|)^{m-k} .
$$

Notice that $\mathscr{S}(\mathbf{R})=\cap S^{m}(\mathbf{R}) . S^{m}(\mathbf{R})$ is a Fréchet space, with the best constants $C_{k}$ as seminorms.

(5.5) Theorem. Let $S$ be a Clifford bundle on the manifold $M$ of bounded geometry with Dirac operator $D$. Let $f \in S^{m}(\mathbf{R})$. Then the operator $f(D)$ (defined via the spectral theorem) is a uniform operator on $S$ of order $\leqslant m$.

Proof. We use the ideas of [11]. Recall that if $f \in \mathscr{S}(\mathbf{R})$, then $f(D)$ is given by the Fourier integral

$$
f(D)=\frac{1}{2 \pi} \int \hat{f}(t) e^{i t D} d t .
$$

In the general case $f \in S^{m}(\mathbf{R})$, let $g \in C_{c}^{\infty}(\mathbf{R})$ with $g(0)=1$. Let $f_{\varepsilon}(x)=$ $f(x) g(\varepsilon x)$. As $\varepsilon \rightarrow 0$, the functions $f_{\varepsilon}$ form a bounded subset of $S^{m}(\mathbf{R})$, as follows directly from Liebniz' rule. Let $s$ be an element of $W^{k}(S)$. Then as $\varepsilon \rightarrow 0, f_{\varepsilon}(D) s$ tends to a limit in $W^{k-m} s$, which limit is $f(D) s$ as defined by the spectral theorem. This is a simple consequence of the dominated convergence theorem.

Now choose $h \in C_{c}^{\infty}(\hat{\mathbf{R}})$ supported with $[-1,1]$ and identically equal to one on $\left[-\frac{1}{2}, \frac{1}{2}\right]$. Let $h_{r}(t)=h(t / r)$ and let $f_{\varepsilon, r}=\left(\mathscr{F}^{-1} h_{r}\right) * f_{\varepsilon}$; here $\mathscr{F}^{-1}$ is the inverse Fourier transform and $*$ denotes convolution.

Suppose that $s$ is an element of $W^{k}(S)$ supported within a set $K$. Then $f_{f, r}(D) s$ is supported within $\operatorname{Pen}^{+}(K, r)$. To see this, write

$$
f_{\varepsilon, r}(D) s=\frac{1}{2 \pi} \int \hat{f}_{\varepsilon}(t) h_{r}(t) e^{i t D} s d t .
$$

Because of the unit propagation speed property (1.3), $e^{i t D_{S}}$ is supported within $\operatorname{Pen}^{+}(K, r)$ for all $t \in[-r, r]$. Since $\operatorname{supp}\left(h_{r}\right) \subset[-r, r]$, the assertion follows.

Therefore,

$$
\left\|f_{\varepsilon}(D) s\right\|_{M \backslash \mathrm{Pen}^{+}(K, r)} \leqslant\left\|\left(f_{\varepsilon}-f_{\varepsilon, r}\right)(D) s\right\|_{k-m} .
$$

The right-hand expression is equal to the norm of

$$
\frac{1}{2 \pi} \int \hat{f}_{\varepsilon}(t)\left(1-h_{r}(t)\right) e^{i t D} s d t
$$

in the space $W^{k-m}(S)$. There is no loss of generality in supposing that $m \leqslant 0$. Thus one has to estimate the $W^{k}$ norms of expressions

$$
\frac{1}{2 \pi} \int \hat{f}_{\varepsilon}(t)\left(1-h_{r}(t)\right) D^{\prime} e^{i t D} s d t
$$


for $0 \leqslant l \leqslant-m$. By definition, these are equal to

$$
\frac{1}{2 \pi} \int \hat{f}_{\varepsilon}(t)\left(1-h_{r}(t)\right)(-i d / d t)^{l}\left(e^{i t D} s\right) d t
$$

Integrating by parts $l$ times, one gets

$$
\frac{1}{2 \pi} \int(i d / d t)^{l}\left[\hat{f}_{\varepsilon}(t)\left(1-h_{r}(t)\right)\right] e^{i t D} s d t
$$

Since $e^{i t D}$ is a unitary operator, this finally gives

$$
\begin{aligned}
\left\|f_{\varepsilon}(D) s\right\|_{k-m, M} & \operatorname{Pen}^{+}(K, r) \\
& \leqslant\|s\|_{k} \cdot \sum_{l}\left\{(1 / 2 \pi) \int\left|(d / d t)^{l}\left[\hat{f}_{\varepsilon}(t)\left(1-h_{r}(t)\right)\right]\right| d t\right\} .
\end{aligned}
$$

Now the functions $f_{\varepsilon}$ form a bounded subset of $S^{m}(\mathbf{R})$. In particular, there is a constant $C$ independent of $\varepsilon$ such that $\left|f_{\varepsilon}^{(j)}(x)\right|<C(1+|x|)^{-2}$ for some $j \geqslant 2$. Therefore

$$
\left|t^{j} \hat{f}_{\varepsilon}(t)\right| \leqslant C \int(1+|x|)^{-2} d x \leqslant 4 C
$$

so that $\left|\hat{f}_{\varepsilon}(t)\right| \leqslant 4 C|t|^{-j}$. Moreover, the functions $x^{l} f_{\varepsilon}(x)$ form a bounded subset of $S^{m+l}(\mathbf{R})$, so the same reasoning may be applied to them to show that

$$
\left|(d / d t)^{\prime} \hat{f}_{\varepsilon}(t)\right| \leqslant C_{l}|t|^{-2}
$$

So by Liebniz' rule

$$
\frac{1}{2 \pi} \int\left|(d / d t)^{l}\left[\hat{f}_{\varepsilon}(t)\left(1-h_{r}(t)\right)\right]\right| d t \rightarrow 0 \quad \text { as } r \rightarrow \infty
$$

and the convergence is uniform in $\varepsilon$. So put

$$
\mu(r)=\sup _{\varepsilon} \sum_{l}\left\{\frac{1}{2 \pi} \int\left|(d / d t)^{l}\left[\hat{f}_{\varepsilon}(t)\left(1-h_{r}(t)\right)\right]\right| d t\right\}
$$

which tends to zero as $r \rightarrow \infty$; we have shown that

$$
\left\|f_{\varepsilon}(D) s\right\|_{k-m, M \backslash \operatorname{Pen}^{+}(K, r)} \leqslant \mu(r)\|s\|_{k} .
$$

Since $\left\|\left(f_{\varepsilon}(D)-f(D)\right) s\right\|_{k-m} \rightarrow 0$ as $\varepsilon \rightarrow 0$, the result follows.

Remark. In the sequel we will make use of the explicit formula (5.6) for $\mu(r)$ derived above.

\section{Regular exhaustions}

As before, let $M$ be a noncompact, oriented, connected Riemannian manifold of bounded geometry. Let $\left(M_{i}\right)$ be an increasing sequence of compact subsets of $M$, whose union is $M$. 
(6.1) Definition. The sequence $\left(M_{i}\right)$ is said to be a regular exhaustion of $M$ if for each $r \geqslant 0$ the quotient

$$
\operatorname{Vol}\left(\operatorname{Pen}^{+}\left(M_{i}, r\right)\right) / \operatorname{Vol}\left(\operatorname{Pen}^{-}\left(M_{i}, r\right)\right)
$$

tends to the limit 1 as $i \rightarrow \infty$.

Of course, it then follows automatically that the quotients

$$
\operatorname{Vol}\left(\operatorname{Pen}{ }^{+}\left(M_{i}, r\right)\right) / \operatorname{Vol}\left(M_{i}\right), \quad \operatorname{Vol}\left(M_{i}\right) / \operatorname{Vol}\left(\operatorname{Pen}^{-}\left(M_{i}, r\right)\right)
$$

also tend to 1 as $i \rightarrow \infty$.

This sort of definition appears to be due originally to Ahlfors [1]. More recently, several authors have considered related ideas, for example [9], [27], [30], [31].

Example. Let $X_{i}$ be the ball of radius $i$ in $\mathbf{R}^{n}$. It is easy to see that the sequence $\left(X_{i}\right)$ is a regular exhaustion.

This example can be generalized. Suppose that there is a point $x_{0}$ in $M$ such that for all $p>0$,

$$
e^{-p r} \operatorname{Vol}\left(B\left(x_{0}, r\right)\right) \rightarrow 0 \text { ad } r \rightarrow \infty ;
$$

then one says that $M$ has subexponential growth.

(6.2) Proposition. If $M$ has subexponential growth, then it has a regular exhaustion.

Proof. Let $B_{j}=B\left(x_{0}, j\right)$ and let $\varepsilon>0$ and an integer $r$ be given. We claim that there are infinitely many indices $j$ for which

$$
\operatorname{Vol}\left(\operatorname{Pen}^{+}\left(B_{j}, r\right)\right) / \operatorname{Vol}\left(\operatorname{Pen}^{-}\left(B_{j}, r\right)\right)<1+\varepsilon .
$$

For suppose the contrary; then for all sufficiently large $j$,

$$
\operatorname{Vol}\left(B_{j+r}\right) \geqslant(1+\varepsilon) \operatorname{Vol}\left(\operatorname{Pen}^{-}\left(B_{j}, r\right)\right) \geqslant(1+\varepsilon) \operatorname{Vol}\left(B_{j-r}\right)
$$

which implies that $\operatorname{Vol}\left(B_{j}\right)$ grows exponentially in $j$, a contradiction. Hence one can choose by an inductive procedure a subsequence $\left(M_{i}\right)$ of the $\left(B_{j}\right)$ such that

$$
\operatorname{Vol}\left(\operatorname{Pen}^{+}\left(M_{i}, i\right)\right) / \operatorname{Vol}\left(\operatorname{Pen}^{-}\left(M_{i}, i\right)\right)<1+1 / i .
$$

Clearly, $\left(M_{i}\right)$ is a regular exhaustion.

These regular exhaustions are related to the "fundamental classes" introduced in $\S 3$. To see this we need good bump functions on $M$.

(6.3) Lemma. Let $L$ be a subset of $M$. Given any $\varepsilon>0$, there is a smooth function $\phi$ on $M$ with values in $[0,1]$ such that:

(i) $\phi=1$ on $L$;

(ii) $\phi=0$ outwith $\operatorname{Pen}^{+}(L, 1 / \varepsilon)$;

(iii) $|\nabla \phi|<2 \varepsilon$. 
Proof. Take $\phi$ to be a smooth approximation to the Lipschitz function $x \rightarrow \max \{0,1-\varepsilon \operatorname{dist}(x, L)\}$.

Recall from $\S 3$ that $\Omega_{\beta}^{n}(M)$ denotes the Banach space of bounded $n$-forms on $M$. An element $m$ of the dual of $\Omega_{\beta}^{n}(M)$ will be said to be associated to a regular exhaustion $\left(M_{i}\right)$ of $M$ if for each bounded $n$-form $\alpha$

$$
\liminf _{i \rightarrow \infty}\left|\langle\alpha, m\rangle-\frac{1}{\operatorname{Vol} M_{i}} \int_{M_{i}} \alpha\right|=0 \text {. }
$$

(6.4) Proposition. There exist functionals associated with every regular exhaustion.

Proof. Let $\left(M_{i}\right)$ be a regular exhaustion of $M$, and let $m_{i}$ be the corresponding functional on $\Omega_{\beta}^{n}$ defined by

$$
\left\langle\alpha, m_{i}\right\rangle=\frac{1}{\operatorname{Vol} M_{i}} \int_{M_{i}} \alpha
$$

These functionals belong to the unit ball of the dual of the Banach space $\Omega_{\beta}^{n}$, which is compact in the weak-star topology by the Banach-Alaoglu theorem [33, Chapter V, Appendix, Theorem 1.1]. Take $m$ to be a weak-star limit point of the infinite set $\left\{m_{i}\right\}$.

(6.5) Proposition. Any functional associated with a regular exhaustion is a fundamental class in the sense of (3.3).

Proof. Let $m$ be associated to a regular exhaustion $\left(M_{i}\right)$, and let $\beta$ be a bounded $(n-1)$-form. One must show that $\langle m, d \beta\rangle=0$.

For each set $M_{i}$, let $\phi_{i}$ be a function on $M$ satisfying the conditions of Lemma 6.3 for $L=M_{i}$ and $\varepsilon=1$. Clearly, for any bounded $n$-form $\alpha$

$$
\left|\int_{M_{i}} \alpha-\int_{M} \alpha \phi_{i}\right| \leqslant\left(\operatorname{Vol}\left(\operatorname{Pen}^{+}\left(M_{i}, 1\right)\right)-\operatorname{Vol}\left(M_{i}\right)\right)\|\alpha\|
$$

and so

$$
\frac{1}{\operatorname{Vol} M_{i}}\left(\int_{M_{i}} \alpha-\int \alpha \phi_{i}\right) \rightarrow 0 \quad \text { as } i \rightarrow \infty .
$$

Thus it suffices to show that

$$
\frac{1}{\operatorname{Vol} M_{i}} \int_{M} \phi_{i} d \beta \rightarrow 0 \quad \text { as } i \rightarrow \infty \text {. }
$$

One may integrate by parts to obtain

$$
\int \phi_{i} d \beta=-\int d \phi_{i} \wedge \beta
$$


Since $\operatorname{supp}\left(d \phi_{i}\right) \subset \operatorname{closure}\left(\operatorname{Pen}^{+}\left(M_{i}, 1\right) \backslash M_{i}\right)$, this implies that

$$
\left|\int \phi_{i} d \beta\right| \leqslant 2\left(\operatorname{Vol}\left(\operatorname{Pen}^{+}\left(M_{i}, 1\right)\right)-\operatorname{Vol}\left(M_{i}\right)\right)\|\beta\|,
$$

and the desired result follows.

Remark. Since $\Omega_{\beta}^{n}(M)$ is not separable unless $M$ is compact, the appeal made above to the Banach-Alaoglu theorem involves the full strength of the axiom of choice.

Some important examples of regularly exhaustible manifolds arise from coverings. Let $X$ be a compact oriented manifold, and let $M$ be a Galois covering of $X$ with Galois group $\Gamma$. If $\Gamma$ is amenable, we will say that $M$ is an amenable covering of $X$.

(6.6) Proposition. Suppose that $M$ is an amenable covering of a compact manifold $X$, equipped with any Riemannian structure pulled back from $X$. Then $M$ has a regular exhaustion with associated fundamiental class $m$ such that for any top-dimensional form $\alpha$ on $X$,

$$
\int_{X} \alpha=\left\langle m, \pi^{*} \alpha\right\rangle
$$

where $\pi: M \rightarrow X$ is the covering projection.

Proof (cf. [9]). We will need to make use of Følner's theorem (see [22]). This gives a necessary and sufficient condition for $\Gamma$ to be amenable, namely that for each $\varepsilon>0$ and each finite subset $\Sigma$ of $\Gamma$ there should be a finite subset $E$ of $\Gamma$ such that

$$
\operatorname{Card}(E \cap \gamma E) \geqslant(1-\varepsilon) \operatorname{Card}(E) \quad \forall \gamma \in \Sigma \text {. }
$$

Let $F$ be a fundamental domain for the action of $\Gamma$ on $M$. Now for $r=1,2, \cdots$ let $\Sigma_{r}$ be the finite subset of $\Gamma$ consisting of those $\gamma$ such that $\operatorname{dist}(F, \gamma F) \leqslant r$. Let $E_{r}$ be the finite subset of $\Gamma$ given by Følner's thoerem such that $\operatorname{Card}\left(E_{r} \cap \gamma E_{r}\right) \geqslant(1-\varepsilon) \operatorname{Card}\left(E_{r}\right) \quad \forall \gamma \in \Sigma_{r}$, where $\varepsilon=$ $1 /\left(r \operatorname{Card}\left(\Sigma_{r}\right)\right)$. Finally, let $M_{r}=\bigcup\left\{\gamma F: \gamma \in E_{r}\right\}$. We claim that $\left(M_{r}\right)$ is a regular exhaustion of $M$.

To see this, assume without loss of generality that $\operatorname{Vol}(F)=1$; then $\operatorname{Vol}\left(M_{r}\right)=\operatorname{Card}\left(E_{r}\right)$. But also,

$$
M_{r} \backslash \operatorname{Pen}^{-}\left(M_{r}, r\right) \subseteq \bigcup\left\{\gamma F: \gamma \in E_{r}, \exists \delta \in \Sigma_{r} \text { s.t. } \gamma \delta^{-1} \notin E_{r}\right\} .
$$

So,

$$
\begin{aligned}
\operatorname{Vol}\left(M_{r} \backslash \operatorname{Pen}^{-}\left(M_{r}, r\right)\right) & \leqslant \sum_{\delta \in \Sigma_{r}} \operatorname{Card}\left(E_{r} \backslash \delta^{-1} E_{r}\right) \\
& \leqslant(1 / r) \operatorname{Card}\left(E_{r}\right) .
\end{aligned}
$$


Similarly, $\operatorname{Vol}\left(\operatorname{Pen}^{+}\left(M_{r}, r\right) \backslash M_{r}\right) \leqslant(1 / r) \operatorname{Card}\left(E_{r}\right)$. Therefore

$$
\left(\operatorname{Vol}\left(\operatorname{Pen}{ }^{+}\left(M_{r}, r\right)\right)-\operatorname{Vol}\left(\operatorname{Pen}{ }^{-}\left(M_{r}, r\right)\right)\right) / \operatorname{Vol}\left(M_{r}\right) \leqslant 2 / r \text {. }
$$

The claim follows.

Let $m$ be a fundamental class on $M$ associated to the regular exhaustion $\left(M_{r}\right)$. Since each of the sets $M_{r}$ is a union of translates of the fundamental domain $F$,

$$
\frac{1}{\operatorname{Vol} M_{i}} \int_{M_{r}} \pi^{*} \alpha=\int_{F} \pi^{*} \alpha=\int_{X} \alpha .
$$

Returning now to the general case, let $m$ be a fundamental class on a manifold $M$ of bounded geometry associated to a regular exhaustion $\left(M_{i}\right)$. Recall from (5.4) that any operator $A$ belonging to the algebra $\mathscr{U}_{-\infty}(M)$ is represented by a uniformly bounded smoothing kernel $k$, so that

$$
A u(x)=\int k(x, y) u(y) \cdot \operatorname{vol}(y)
$$

The $n$-form $\alpha: x \rightarrow k(x, x) \operatorname{vol}(x)$ is therefore bounded. We define $\tau(A)=$ $\langle m, \alpha\rangle$, so that $\tau$ becomes a linear functional on $\mathscr{U}_{-\infty}(M)$.

(6.7) Theorem. The functional $\tau$ thus defined is a trace on the algebra $\mathscr{U}_{-\infty}(M)$.

Proof. Let $A_{1}$ and $A_{2}$ belong to $\mathscr{U}_{-\infty}(M)$, with corresponding kernels $k_{1}$ and $k_{2}$. Let $\varepsilon>0$ be given.

By (5.4), one can choose $r>0$ so large that

$$
\int_{M \backslash B(x, r)}\left|k_{j}(x, y)\right|^{2} \cdot \operatorname{vol}(y)<\varepsilon, \quad \int_{M \backslash B(x, r)}\left|k_{j}(y, x)\right|^{2} \cdot \operatorname{vol}(y)<\varepsilon
$$

for $j=1,2$ and all $x \in M$. From (5.1), there is an upper bound $V$ for the volume of any ball of radius $r$ in $M$. Also, by definition of a regular exhaustion, the inequality

$$
\begin{aligned}
\left(\operatorname{Vol}\left(\operatorname{Pen}^{+}\left(M_{i}, r\right)\right)-\right. & \left.\operatorname{Vol}\left(\operatorname{Pen}^{-}\left(M_{i}, r\right)\right)\right) / \operatorname{Vol}\left(M_{i}\right) \\
& <\varepsilon /\left(V \cdot\left(\sup \left|k_{1}\right|\right) \cdot\left(\sup \left|k_{2}\right|\right)\right)
\end{aligned}
$$

must hold for all sufficiently large $i$.

Let $k$ be the kernel of $A_{1} A_{2}-A_{2} A_{1}$; that is,

$$
k(x, z)=\int_{M}\left(k_{1}(x, y) k_{2}(y, z)-k_{2}(x, y) k_{1}(y, z)\right) \operatorname{vol}(y) \text {. }
$$

Therefore, $\int_{M_{1}} k(x, x) \operatorname{vol}(x)$ is equal to

$$
\iint\left(k_{1}(x, y) k_{2}(y, x)-k_{2}(x, y) k_{1}(y, x)\right) \cdot \operatorname{vol}(x) \operatorname{vol}(y),
$$


where the double integral is taken over the range $\left\{x \in M_{i}, y \in M\right\}$. The absolute value of the double integral will be estimated by dividing this range into three parts:

(i) Over the range $\left\{x \in M_{i}, y \in M_{i}\right\}$ the integral vanishes by symmetry.

(ii) Over the range $\left\{x \in M_{i}, y \in M \backslash M_{i}\right.$, $\left.\operatorname{dist}(x, y)<r\right\}$ both $x$ and $y$ belong to $\operatorname{Pen}^{+}\left(M_{i}, r\right) \backslash \operatorname{Pen}^{-}\left(M_{i}, r\right)$. For fixed $x$, the modulus of the integral with respect to $y$ is bounded by $2 V \sup \left|k_{1}\right| \sup \left|k_{2}\right|$. Thus the modulus of the double integral over this range is bounded by

$$
2 V \sup \left|k_{1}\right| \sup \left|k_{2}\right|\left(\operatorname{Vol}\left(\operatorname{Pen}^{+}\left(M_{i}, r\right)\right)-\operatorname{Vol}\left(\operatorname{Pen}^{-}\left(M_{i}, r\right)\right)\right)
$$

and, as remarked above, this is less than $2 \varepsilon \operatorname{Vol}\left(M_{i}\right)$ for sufficiently large $i$.

(iii) Over the range $\left\{x \in M_{i}, y \in M \backslash M_{i}\right.$, $\left.\operatorname{dist}(x, y) \geqslant r\right\}$ it follows from the Cauchy-Schwarz inequality and the definition of $r$ that the modulus of the integral with respect to $y$ is less than $2 \varepsilon$, so that the modulus of the double integral over this range is bounded by $2 \varepsilon \mathrm{Vol}\left(M_{i}\right)$.

Thus we have shown that for sufficiently large $i$,

$$
\left|\int_{M_{i}} k(x, x) \cdot \operatorname{Vol}(x)\right| \leqslant 4 \varepsilon \operatorname{Vol}\left(M_{i}\right) \text {. }
$$

But $\varepsilon$ is arbitrary, and

$$
\lim \inf \left(1 / \operatorname{Vol} M_{i}\right)\left|\tau\left(A_{1} A_{2}-A_{2} A_{1}\right)-\int_{M_{i}} k(x, x) \cdot \operatorname{vol}(x)\right|=0 .
$$

It follows that $\tau\left(A_{1} A_{2}-A_{2} A_{1}\right)=0$, as required.

Notice that an analogous functional $\tau$ can be defined on the algebra $\mathscr{U}_{-\infty}(S)$ for any Clifford bundle $S$ by the formula

$$
\tau(A)=m(x \rightarrow \operatorname{Tr} k(x, x))
$$

where $T r$ denotes the pointwise trace on sections of $S \otimes S$. In fact an argument analogous to the previous one shows that this $\tau$ is also a trace, but this will not be needed.

\section{Good embedding}

It will be shown next that the index of the Dirac operator of a Clifford bundle on a manifold $M$ of bounded geometry belongs to $K\left(\mathscr{U}_{-\infty}(M)\right)$. To do this it is necessary to embed the Clifford bundle in a trivial bundle in a good way. We will make use of a simple fact from graph theory to obtain such embeddings: 
(7.1) Lemma. Let $G$ be a (possibly infinite) graph which has finite vertex degree $k$ (that is, at most $k$ edges meet at any one vertex). Then the vertices of $G$ may be colored using $k+1$ colors in such a way that no two vertices of the same color are joined by an edge.

Proof. Left to the reader.

Recall that the order of a covering of a topological space is the least integer $k$ such that the intersection of any $(k+1)$ distinct sets of the covering is empty; if there is no such $k$, one says that the covering has infinite order.

(7.2) Lemma. For any $r>0$, the manifold $M$ has a covering by open balls

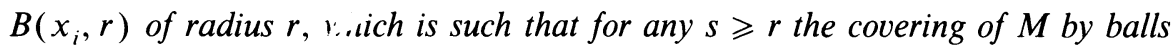
$B\left(x_{i}, s\right)$ has finite order.

Proof. Choose a covering of $M$ by open balls $B\left(x_{i}, r\right)$ in such a way that distinct centers $\left(x_{i}\right)$ are separated by a distance of at least $r$; such coverings may easily be constructed by induction. The balls $B\left(x_{i}, r / 3\right)$ are then pairwise disjoint. If a point $x$ belongs to $B\left(x_{i}, s\right)$, then $B\left(x_{i}, r / 3\right) \subset B(x, s+r / 3)$. Now let $V_{0}$ and $V_{1}$ be the functions defined in Proposition 5.1. The volume of a ball of radius $r / 3$ is at least $V_{0}(r / 3)$, and the volume of a ball of radius $s+r / 3$ is at most $V_{1}(s+r / 3)$. Thus a point $x$ can belong to at most $V_{1}(s+r / 3) / V_{0}(r / 3)$ of the balls $B\left(x_{i}, s\right)$, which is what was required.

(7.3) Lemma. For any $r>0$, the manifold $M$ has a finite covering by open sets, each of which is a disjoint union of balls of radius $r$ whose centers are separated by a distance of at least $4 r$.

Proof. From a covering $\left\{B\left(x_{i}, r\right)\right\}$ satisfying the conditions of (7.2), construct a graph $G$ as follows: the vertices of $G$ correspond to the points $x_{i}$, and two vertices are linked by an edge if and only if the corresponding points are separated by a distance of less than $4 r$. By (7.2), the covering $\left\{B\left(x_{i}, 4 r\right)\right\}$ of $M$ has finite order, which implies that $G$ has finite vertex-degree. Color the vertices of $G$ with finitely many colors according to (7.1). If $U_{l}$ denotes the union of all the $B\left(x_{i}, r\right)$ for which the vertex corresponding to $x_{i}$ is colored $l$, then the covering $\left\{U_{1}\right\}$ satisfies the required conditions.

Let $S_{0}$ and $S_{1}$ be (Clifford) bundles of bounded geometry on $M$, and let $\iota$ : $S_{0} \rightarrow S_{1}$ be an isometric inclusion. (Note that $\iota$ is not assumed to be a module-homomorphism. We are using the Clifford bundle structure here merely as a crutch to define Sobolev spaces and hence uniform operators.) There is a natural orthogonal projection $\pi: S_{1} \rightarrow S_{0}$ which is a left inverse for $\iota$. The inclusion $\iota$ will be called good if both $\iota$ and $\pi$ are uniform operators of order $\leqslant 0$.

Recall that $\mathscr{U}_{-\infty}(S)$ is an ideal in the algebra $\mathscr{U}(S)$. We shall be interested in abstract elliptic operators (in the sense of $\S 4$ ) relative to this ideal. Recall that such an operator has an index in $K\left(\mathscr{U}_{-\infty}(S)\right)$. 
Now suppose that $\iota: S_{0} \rightarrow S_{1}$ is a good inclusion. Then the map $\iota_{*}: \rho \rightarrow \iota \rho \pi$ is a homomorphism of algebras $\mathscr{U}_{-\infty}\left(S_{0}\right) \rightarrow \mathscr{U}_{-\infty}\left(S_{1}\right)$, and so induces a homomorphism (which will also be denoted $\left.\iota_{*}\right)$ from $K\left(\mathscr{U}_{-\infty}\left(S_{0}\right)\right.$ ) to $K\left(\mathscr{U}_{-\infty}\left(S_{1}\right)\right)$.

(7.4) Proposition. Let $P$ be an abstract elliptic operator on $S_{0}$. Then $\iota_{*}($ Ind $P) \in K\left(\mathscr{U}_{-\infty}\left(S_{1}\right)\right)$ is independent of the good inclusion $\iota$.

Proof. Let $P: M \rightarrow N$ be an abstract elliptic operator between finite projective $\mathscr{U}\left(S_{0}\right)$-modules. For brevity, write $A$ for the algebra $\mathscr{U}\left(S_{1}\right)$. Then $\iota_{*} P$ is abstractly elliptic between the $A$-modules $M \otimes_{\iota_{*}} A$ and $N \otimes_{\iota_{*}} A$. By naturality, $\iota_{*}(\operatorname{Ind} P)=\operatorname{Ind}\left(\iota_{*} P\right)$ in $K\left(\mathscr{U}_{-\infty}\left(S_{1}\right)\right)$. Now let $\iota^{\prime}$ be another good inclusion, with corresponding projection $\pi^{\prime}$. Then there is a commutative diagram

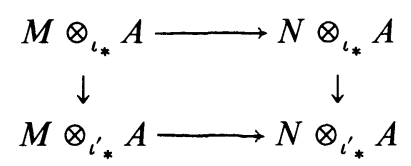

in which the horizontal arrows are the maps $\iota_{*} P, \iota_{*}^{\prime} P$ and the vertical arrows are isomorphisms given by $x \otimes a \rightarrow x \otimes \iota^{\prime} \pi a$. (The inverse isomorphisms are given by $x \otimes a \rightarrow x \otimes \imath \pi^{\prime} a$.) Thus $\operatorname{Ind}\left(\iota_{*} P\right)=\operatorname{Ind}\left(\iota_{*}^{\prime} P\right)$.

(7.5) Proposition. Any Clifford bundle $S$ of bounded geometry has a good inclusion in a trivial bundle.

Sketch of Proof. If $B \subset M$ is a good coordinate ball of radius $r$, then using normal coordinates we may construct on $B$ an isomorphism of $S_{\mid B}$ with a trivial bundle $B \times C^{k}$. This isomorphism and its inverse are by (2.4) and (2.5) uniform operators of order 0 . So, by (7.3) we can find a finite covering of $M$ by open sets that are unions of such balls $B$, over each of which $S$ is uniformly isomorphic to a $k$-dimensional trivial bundle. Now by taking the direct sum and using a partition of unity (as in the analogous construction for compact manifolds, cf. [26, Chapter $3, \S 5]$ ) we can obtain a good inclusion of $S$ in an $m k$-dimensional trivial bundle.

Let $P$ be an abstractly elliptic operator on $S$. By (7.5) there exists a good inclusion $\iota$ of $S$ in a trivial bundle $T$, and $\iota_{*}($ Ind $P) \in K\left(\mathscr{U}_{-\infty}(T)\right)$ is independent of the choice of $\iota$. However, $K\left(\mathscr{U}_{-\infty}(T)\right)$ is isomorphic to $K\left(\mathscr{U}_{-\infty}(M)\right)$ by (4.2), so that we obtain a well-defined element, also denoted $\operatorname{Ind}(P)$, of $K\left(\mathscr{U}_{-\infty}(M)\right)$.

Now, let $D$ be the Dirac operator on the Clifford bundle $S$, equipped with a grading $\eta$.

(7.6) Lemma. The operator $D$ is abstractly elliptic between the $\mathscr{U}(S)$-modules given by the eigenprojections $(1+\eta) / 2$ and $(1-\eta) / 2$.

(These modules are of course the "positive and negative spin-spaces.") 
Proof. Let $\phi$ be a compactly supported smooth function on $\mathbf{R}$ equal to one in a neighborhood of zero. Let $\psi(x)=\left(1-\phi\left(x^{2}\right)\right) / x^{2}$. Then $\psi$ belongs to the symbol class $S^{-2}(\mathbf{R})$, so that $\psi(D) \in \mathscr{U}_{-2}(S)$ by (5.5). Hence $D \psi(D) \in$ $\mathscr{U}_{-1}(S)$. Now consider the equation

$$
D^{2} \psi(D)=1-\phi\left(D^{2}\right) .
$$

By (5.5), $\phi\left(D^{2}\right)$ is a uniform operator of order $-\infty$, and it commutes with $\eta$. Moreover, $D \psi(D)$ anticommutes with $\eta$. It follows that $D \psi(D)$ is a parametrix for $D$.

The Dirac operator $D$ therefore has an index $\operatorname{Ind}(D)$ in $K\left(\mathscr{U}_{-\infty}(M)\right)$.

\section{The main theorem}

Let $M$ and $S$ be of bounded geometry, $\eta$ a grading, and $D$ the Dirac operator. Suppose that $M$ admits a regular exhaustion with corresponding fundamental class $m$ and trace $\tau$ on $\mathscr{U}_{-\infty}(M)$. In the previous section we have defined the index $\operatorname{Ind}(D)$ in $K\left(\mathscr{U}_{-\infty}(M)\right)$. But the results of $\S 4$ show that there is a dimension homomorphism $\operatorname{dim}_{\tau}: K\left(\mathscr{U}_{-\infty}(M)\right) \rightarrow \mathbf{R}$. We must now compute $\operatorname{dim}_{\tau}($ Ind $D)$.

(8.1) Proposition. Let $f$ be any Schwartz-class function on $\mathbf{R}^{+}$with $f(0)=1$. Then

$$
\operatorname{dim}_{\tau}(\operatorname{Ind} D)=\tau\left(\eta f\left(D^{2}\right)\right) .
$$

(The definition of $\tau$ on $\mathscr{U}_{-\infty}(S)$ was given at the end of $\S 6$.)

Proof. First consider the special case $f(\lambda)=\phi(\lambda)^{2}, \phi$ being a function of the sort considered in (7.6) above. Then the result follows from (4.1); the only thing to be checked is that the abstract extension of $\tau$ to the projective modules $(1+\eta) / 2$ and $(1-\eta) / 2$ described in $\S 4$ is the same as the concrete extension of $\tau$ to $\mathscr{U}_{-\infty}(S)$ described at the end of $\S 6$. Next, suppose that $f(\lambda)=\phi(\lambda)^{2}$, where $\phi$ is now any smooth function of compact support with $\phi(0)=1$. Then $\phi$ may be approximated smoothly by a sequence $\phi_{j}$ of functions of fixed compact support that are equal to one in a neighborhood of zero. By (2.10), the Schwartz kernels of $\phi_{j}\left(D^{2}\right)^{2}$ tend uniformly to the Schwartz kernel of $f\left(D^{2}\right)$ as $j \rightarrow \infty$. So

$$
\tau\left(\eta f\left(D^{2}\right)\right)=\lim \tau\left(\eta \phi_{j}\left(D^{2}\right)^{2}\right)=\operatorname{dim}_{\tau}(\text { Ind } D) .
$$

We deduce by linearity and continuity the more general formula

$$
\tau\left(\eta \phi\left(D^{2}\right)^{2}\right)=\phi(0)^{2} \operatorname{dim}_{\tau}(\text { Ind } D)
$$

valid for any smooth $\phi$ of compact support. Since the linear span of $\left\{\phi^{2}\right.$ : $\left.\phi \in C_{c}^{\infty}(\mathbf{R})\right\}$ is dense in $\mathscr{R}(\mathbf{R})$, the general result follows by (2.10). 
Now take $f(\lambda)=e^{-t \lambda}$, and apply the asymptotic expansion theorem (2.11). This gives the asymptotic expansion as $t \rightarrow 0$

$$
\operatorname{dim}_{\tau}\left(\text { Ind } D^{+}\right) \sim \sum t^{(k-n) / 2}\left\langle\operatorname{Tr}\left(\eta_{k}^{\Psi}\right), m\right\rangle .
$$

Since the left-hand side is independent of $t$, only the constant term can appear on the right-hand side. By definition, $\operatorname{Tr}\left(\eta_{n}^{\Psi}\right)$ is the index form $\mathbf{I}(D)$, so that we have completed the proof of

(8.2) Index Theorem. Let $M$ be a Riemannian manifold and $S$ a graded Clifford bundle on $M$, both with bounded geometry. Let $D$ be the Dirac operator of $S$. Let $m$ be a fundamental class for $M$ associated to a regular exhaustion, and let $\tau$ be the corresponding trace on the algebra of uniform operators of order $-\infty$. Then $D$ is abstractly elliptic on $S$, and

$$
\operatorname{dim}_{\tau}(\operatorname{Ind} D)=\langle\mathbf{I}(D), m\rangle
$$

where $\mathbf{I}(D)$ is the usual integrand in the Atiyah-Singer formula for $D$.

Remarks. (a) The index-form $\mathbf{I}(D)$ is given by a characteristic class formula, and is therefore an element of the $\beta$-cohomology group $H_{\beta}^{n}(M)$. Thus the right-hand side of the formula is cohomological in nature. In particular (see 3.8) the index remains unchanged under strict quasi-isometry of the metric on $M$.

(b) We could have reached this formula a little more quickly had we been prepared to construct $\operatorname{Ind}(D)$ as an element of $K\left(\mathscr{U}_{-\infty}(S)\right)$ rather than of $K\left(\mathscr{U}_{-\infty}(M)\right)$. The apparatus of "good embedding" would then have been unnecessary. However we preferred to show how $K\left(\mathscr{U}_{-\infty}(M)\right)$ is a "universal" receptacle for indices of elliptic operators. This prepares the way for the possible application of more advanced $K$-theoretic techniques, such as the cyclic cohomology theory of A. Connes [16].

\section{References}

[1] L. V. Ahlfors, Zur Theorie der Uberlagerungsflachen, Acta Math. 65 (1935) 157-194.

[2] L. V. Ahlfors \& L. Sario, Riemann surfaces, Frinceton University Press, Princeton, NJ, 1960.

[3] M. F. Atiyah, Elliptic operators, discrete groups, and von Neumann algebras, Asterisque 32 (1976) 43-72.

[4] M. F. Atiyah, R. Bott \& V. K. Patodi, On the heat equation and the index theorem, Invent. Math. 19 (1973) 279-330.

[5] M. F. Atiyah, H. Donnelley \& I. M. Singer, Eta invariants, signature defects of cusps, and values of L-functions, Ann. of Math. (2) 118 (1983) 131-177.

[6] M. F. Atiyah, V. K. Patodi \& I. M. Singer, Spectral asymmetry and Riemannian geometry. I, Math. Proc. Cambridge Philos. Soc. 77 (1975) 43-69.

[7] M. F. Atiyah \& W. Schmid, A geometric construction of the discrete series for semi-simple Lie groups, Invent. Math. 42 (1977) 1-62.

[8] M. F. Atiyah \& I. M. Singer, The index of elliptic operators. III, Ann. of Math. (2) 87 (1968) $546-604$ 
[9] R. Brooks, The fundamental group and the spectrum of the Laplacian, Comment. Math. Helv. 56 (1981) 581-598.

[10] J. Cheeger \& M. Gromov, Bounds on the von Neumann dimension of $L^{2}$-cohomology and the Gauss-Bonnet theorem for open manifolds, J. Differential Geometry 21 (1985) 1-34.

[11] J. Cheeger, M. Gromov \& M. Taylor, Finite propagation speed, kernel estimates for functions of the Laplace operator, and the geometry of complete Riemannian manifolds, J. Differential Geometry 17 (1982) 15-54.

[12] P. R. Chernoff, Essential self-adjointness of powers of generators of hyperbolic equations, J. Functional Analysis 12 (1973) 401-414.

[13] L. A. Coburn, R. G. Douglas, D. G. Schaeffer \& I. M. Singer, $C^{*}$-algebras of operators on a half-space. II: Index theory, Inst. Hautes Études Sci. Publ. Math. 40 (1971) 69-80.

[14] A. Connes, Sur la theorie non-commutative de l'integration, Lecture Notes in Math. Vol. 725 , Springer, Berlin, 1979, 19-143.

[15] _ A survey of foliations and operator algebras; Proc. Sympos. Pure Math., No. 38, Amer. Math. Soc., Providence, RI, 1982, 521-628.

[16] _ Non-commutative differential geometry, Publ. Math. Inst. Hautes Études Sci. 62 (1985) 41-144.

[17] A. Connes \& H. Moscovici, The $L^{2}$-index theorem for homogeneous spaces of Lie groups, Ann. of Math. (2) 115 (1982) 291-330.

[18] A. Connes \& G. Skandalis, The longitudinal index theorem for foliations, Publ. Res. Inst. Math. Soc. 20 (1984) 1139-1183.

[19] J. Dieudonné, Elements d' analyse, nine volumes, Gauthier-Villars, Paris, 1961-1982.

[20] J. Dodziuk, $L^{2}$ harmonic forms on complete manifolds, in Seminar on Differential Geometry (S.-T. Yau, ed.), Annals of Math. Studies, No. 102, Princeton University Press, Princeton, NJ, 1982, 291-302.

[21] P. B. Gilkey, The heat equation and the index theorem, Publish or Perish, Boston, 1977.

[22] F. P. Greenleaf, Invariant means on topological groups, Van Nostrand Math. Studies, No. 16, 1969.

[23] M. Gromov \& B. Lawson, Positive scalar curvature and the Dirac operator, Inst. Hautes Études Sci. Publ. Math. 58 (1983) 83-196.

[24] G. Harder, A Gauss-Bonnet formula for discrete arithmetically defined groups, Ann. Sci. Éole Norm. Sup. (4) 4 (1971) 409-455.

[25] L. Hormander, The Weyl calculus of pseudo-differential operators, Comm. Pure Appl. Math. 32 (1979) 359-443.

[26] D. Husemoller, Fibre bundles, 2nd ed., Springer, Berlin, 1975.

[27] T. Januszkiewicz, Characteristic invariants of non-compact Riemannian manifolds, Topology 23 (1984) 289-302.

[28] S. Kobayashi \& Nomizu, Foundations of differential geometry, Interscience, New York, 1963 and 1969.

[29] J. Milnor, Introduction to algebraic K-theory, Princeton University Press, Princeton, NJ, 1971.

[30] J. Plante, Foliations with measure-preserving holonomy, Ann. of Math. (2) 102 (1975) 327-361.

[31] D. Sullivan, Cycles for the dynamical study of foliated manifolds and complex manifolds, Invent. Math. 36 (1976) 225-255.

[32] M. Taylor, Pseudo-differential operators, Princeton University Press, Princeton, NJ, 1982.

[33] K. Yosida, Functional analysis, 6th ed., Springer, Berlin, 1980.

Jesus COllege, OXford 
